\title{
OATP1B3 Expression and Function is Modulated by Coexpression with OCT1, OATP1B1, and NTCP
}

\author{
Yuchen Zhang, Melissa Ruggiero, and @Bruno Hagenbuch \\ Department of Pharmacology, Toxicology, and Therapeutics, The University of Kansas Medical Center, Kansas City, Kansas
}

Received October 27, 2019; accepted May 13, 2020

\begin{abstract}
Organic anion transporting polypeptide (OATP) 1B3 is a drug transporter expressed at the basolateral membrane of human hepatocytes. Along with other transporters, including OATP1B1, $\mathrm{Na}^{+}$/taurocholate cotransporting polypeptide (NTCP), and organic cation transporter (OCT) 1 , it is responsible for the uptake of endo- and xenobiotics into hepatocytes. Our previous studies demonstrated that OATP1B3 can form hetero-oligomers with OATP1B1 in human embryonic kidney 293T (HEK293) cells and with NTCP in both HEK293 cells and frozen human liver sections. To further characterize the hetero-oligomerization of OATP1B3, we investigated OCT1 as a potential interacting partner and determined the functional consequences of OATP1B3 heterooligomerization. We demonstrated interactions between OATP1B3 and OCT1 by coimmunoprecipitation with an anti-OATP1B3 antibody from human hepatocytes. In addition, we visualized the interaction using the proximity ligation assay in both HEK293 cells and in frozen human liver sections. We investigated the functional consequences of OATP1B3 hetero-oligomerization by measuring the OATP1B3 plasma membrane expression and the uptake of the OATP1B3 selective substrate cholecystokinin-8 (CCK-8) in the absence and presence of OATP1B1, NTCP, and OCT1. A significant decrease of OATP1B3 plasma membrane
\end{abstract}

\section{Introduction}

Uptake of endo- and xenobiotics into human hepatocytes is mediated by several drug uptake transporters that are expressed at the sinusoidal membrane. These include the organic anion transporting polypeptide (OATP) 1B1 and OATP1B3 (Hagenbuch and Stieger, 2013), the organic cation transporter (OCT) 1 (Koepsell, 2013), and the $\mathrm{Na}^{+} /$taurocholate cotransporting polypeptide (NTCP) (Claro da Silva et al., 2013). OATP1B1 and OATP1B3 are polyspecific transporters that can transport different endogenous compounds (Stieger and Hagenbuch, 2014; Bednarczyk and Boiselle, 2016) and mediate the uptake of numerous drugs into human hepatocytes (König, 2011; Roth et al., 2012; Hagenbuch and Stieger, 2013). When testing for potential drug-drug interactions the model substrates estradiol-17 $\beta$-glucuronide, estrone- 3 -

This work was supported by National Institute of General Medical Sciences [R01GM077336], [P20GM103549], and [P30GM118247].

https://doi.org/10.1124/dmd.119.089847. expression was observed after coexpression with OCT1, whereas coexpression with OATP1B1 or NTCP resulted in an increase of plasma membrane expression. With respect to transport, coexpression of OCT1 increased the apparent turnover rate of OATP1B3, whereas coexpression of OATP1B1 or NTCP decreased it. These findings demonstrated that coexpression of OATP1B3 with OATP1B1, NTCP, and OCT1 in HEK293 cells results in a transporter-dependent modification of OATP1B3mediated CCK-8 transport and suggest that functional results obtained in single transporter overexpressing cell lines over- or underestimate OATP1B3 function in human hepatocytes.

\section{SIGNIFICANCE STATEMENT}

Coexpression of organic anion transporting polypeptide (OATP) 1B3 with organic cation transporter (OCT) $1, \mathrm{Na}^{+}$/taurocholate cotransporting polypeptide, or OATP1B1 in human embryonic kidney 293T cells affects its expression level and function. When OCT1 is knocked down in human hepatocytes, function of OATP1B3 goes up. These results suggest that protein-protein interactions can affect the expression and function of the involved proteins, and thus single transporter expression systems might lead to over- or underestimation of drug-drug interactions.

ABBREVIATIONS: ASBT, apical sodium-dependent bile acid transporter; CCK-8, cholecystokinin-8; DAPI, 4',6-diamidino-2-phenylindole; DTSSP, 3,3'-dithiobis(sulfosuccinimidyl proprionate); EV, empty vector; HEK293, human embryonic kidney 293T; MPP ${ }^{+}$, 1-methyl-4-phenylpyridinium; NHE3, epithelial sodium-proton exchanger; NTCP, $\mathrm{Na}^{+} /$taurocholate cotransporting polypeptide; OATP, organic anion transporting polypeptide; OCT, organic cation transporter; shRNA, small hairpin RNA. 
A

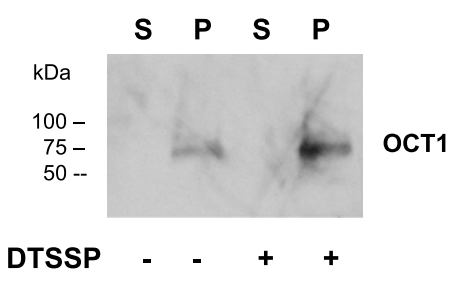

B

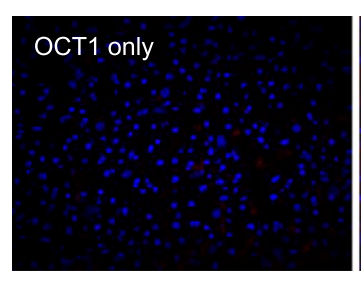

Fig. 1. OATP1B3 and OCT1 interact in human hepatocytes. Interaction of OATP1B3 with OCT1 is demonstrated by (A) coimmunoprecipitation and (B) with a proximity ligation assay. (A) Solubilized proteins from freshly isolated and plated human hepatocytes were incubated with an anti-OATP1B3 antibody (400B3) in the presence or absence of the crosslinking agent DTSSP. After precipitating the anti-OATP1B3 antibodies with protein A beads, the resulting supernatants (S) and pellets (P) were separated by SDS-PAGE, transferred to a polyvinylidene fluoride membrane, and incubated with an anti-OCT1 antibody. (B) Frozen human liver sections were incubated either with an anti-OCT1 antibody alone or with a combination of anti-OCT1 and anti-OATP1B3 (K28) antibodies. After incubation with the respective secondary antibodies and the ligation reaction mix, interactions can be detected in red. The blue color represents DAPI staining of the nuclei.
NTCP (Zhang et al., 2017). In addition, a modified yeast two-hybrid screening known as DUALmembrane screening (Stagljar et al., 1998) identified OCT1 as a likely interaction partner. However, the functional consequences of these hetero-oligomers remain unknown. Given that the Food and Drug Administration guidelines for the study of transporter-mediated drug-drug interactions include OATP1B3, OATP1B1, and OCT1 as potential targets for DDIs, it is important to understand the influence of the hetero-oligomerization of OATP1B3 with these three transporters. Therefore, in the present study we investigated whether OATP1B3 and OCT1 indeed interact with each other, and we characterized the functional consequences of coexpression of OATP1B3 with OCT1, OATP1B1, or NTCP in human embryonic kidney 293T (HEK293) cells. Furthermore, we determined the consequences of OCT1 knockdown in primary human hepatocytes on OATP1B3 function using small hairpin RNA (shRNA) experiments.

\section{Materials and Methods}

Materials. Frozen human liver was obtained in accordance with a protocol approved by the Institutional Review Board of the University of Kansas Medical Center from patients undergoing hepatic resection procedures or from liver donors through the Cell Isolation Core in the Department of Pharmacology, Toxicology, and Therapeutics at the University of Kansas Medical Center. Cryopreserved human hepatocytes (HUM4180A, from a 7-year-old white male) were purchased from Lonza (Walkersville, MD). Freshly isolated human hepatocytes (KHH134 from a 43-year-old white male and KHH135 from a 61-year-old white female) were obtained from the Cell Isolation Core of the department of Pharmacology, Toxicology and Therapeutics at the University of Kansas Medical Center. Radiolabeled $\left[{ }^{3} \mathrm{H}\right] \mathrm{CCK}-8(87.9 \mathrm{Ci} / \mathrm{mmol})$ and $\left[{ }^{3} \mathrm{H}\right] \mathrm{taur}-$ ocholic acid $(15.4 \mathrm{Ci} / \mathrm{mmol})$ were purchased from PerkinElmer (Boston, MA). $\left[{ }^{14} \mathrm{C}\right]$ metformin $(112 \mathrm{mCi} / \mathrm{mmol})$ was obtained from Moraveck Biochemicals (Brea, CA). Unlabeled CCK-8 was purchased from Bachem (Torrance, CA). HEK293 cells were purchased from American Type Culture Collection (Manassas, VA). Duolink components for the proximity ligation assay were from Sigma-Aldrich (St. Louis, MO). The anti-OCT1 adenovirus against the targeting sequence GCATCGTCTTCCTGGGTTTCA was purchased from Vector Biolabs (Malvern, PA). The Pierce BCA Protein Assay Kit was purchased from ThermoFisher (Waltham, MA). The following antibodies were used: mouse anti-OCT1 antibody purchased from Novus (NBP1-51684; Littleton, CO); mouse anti-FLAG antibody (F3040) and rabbit anti-OATP1B3 antibody (400B3, raised against the C-terminal 12 amino acids) from Sigma-Aldrich; the rabbit anti-OATP1B3 antibody (K28) was a generous gift from Dr. Bruno Stieger (University Hospital Zurich, Switzerland); rabbit anti-His antibody (ab125262) and mouse anti- $\mathrm{Na}^{+} / \mathrm{K}^{+}$ATPase antibody (ab7671) were obtained from Abcam (Cambridge, MA).

DUALmembrane Screening. The DUALmembrane screening (Stagljar et al., 1998), a modified yeast two-hybrid screening, was performed by Dualsystems
Biotech AG (Schlieren, Switzerland) with full-length OATP1B3 as the bait and a human adult liver cDNA library with $1.5 \times 10^{6}$ independent clones.

Cell Culture, Transporter Expression and Uptake Experiments. HEK293 cells were grown in high glucose Dulbecco's modified Eagle's medium (ATCC) supplemented with $10 \%$ fetal bovine serum (Hyclone, Logan, UT), $100 \mathrm{U} / \mathrm{ml}$ penicillin, and $100 \mu \mathrm{g} / \mathrm{ml}$ streptomycin (Invitrogen) in a humidified environment at $37^{\circ} \mathrm{C}$ and $5 \% \mathrm{CO}_{2}$. The open reading frame of OCT1 (NM_003057.2) was polymerase chain reaction-amplified and cloned into the expression plasmid pcDNA5/FRT. The insert of the resulting plasmid was sequenced to verify that the correct sequence was cloned. Construction of His- and FLAG-tagged OATP1B3 and apical sodium-dependent bile acid transporter (ASBT) was described previously (Gui et al., 2008; Zhao et al., 2015; Zhang et al., 2017). Plating, transfection, and uptake experiments on 24-well plates were performed as previously reported (Zhao et al., 2015). Transfection conditions varied among different experiments (for detailed conditions, see figure legends), but in general a constant total amount of $500 \mathrm{ng}$ plasmids was transfected. The following day, the medium was changed, and uptake experiments were performed either 24 or 48 hours after transfections (for time points, see figure legends). Each transfection condition was performed in triplicate.

Cryopreserved human hepatocytes were thawed and plated following the protocols supplied by Thermo Fisher with minor modifications. After thawing, hepatocytes were resuspended in Cryopreserved Hepatocyte Recovery Medium (CM7000; Thermo Fisher) and centrifuged at $100 \mathrm{~g}$ for 10 minutes. The pellet was resuspended in hepatocyte medium [HCM BulletKit (CC-3198; Lonza)] and plated at 400,000 cells per well on collagen-coated 12-well plates. After an overnight culture the cells were treated with adenoviral vectors containing either nontarget control (scrambled) or OCT1-shRNA (multiplicity of infection of 10). After 6 hours of culture the medium was replaced, and the cells were overlaid with Geltrex $(0.35 \mathrm{mg} / \mathrm{ml})$ in the presence of the same concentrations of adenovirus. The medium was then changed daily without additional adenovirus, and on day 6 the medium contained again $0.35 \mathrm{mg} / \mathrm{ml}$ Geltrex. After 11 days of culture uptake was measured, and a membrane enriched fraction was isolated for Western blotting.

Freshly isolate human hepatocytes were plated on collagen coated 12-well plates with 600,000 cells per well from the Cell Isolation Core. After an overnight culture they were treated like the cryopreserved hepatocytes with the exception that Matrigel was used instead of Geltrex. After 11 days in culture the cells were used for uptake experiments, and a membrane enriched fraction was isolated for Western blotting. Because uptake in human hepatocytes cannot be corrected with an empty vector (EV) control, we used accepted transport inhibitors. We measured uptake of CCK- 8 and taurocholate in the absence and presence of $100 \mu \mathrm{M}$ bromosulfophthalein (a well accepted OATP inhibitor) and uptake of $\mathrm{MPP}^{+}$in the absence and presence of $100 \mu \mathrm{M}$ quinidine (a well accepted inhibitor of OCT1). Uptake in the presence of the inhibitor was subtracted from uptake in the absence of inhibitor and was considered transporter-mediated uptake.

Isolation of a Membrane Enriched Fraction. Hepatocytes were washed with $1 \mathrm{ml}$ of PBS and then scraped from 10 (cryopreserved) or from six (freshly isolated hepatocytes) wells into homogenization buffer that consisted of $1 \mathrm{mM}$ $\mathrm{NaCl}, 5 \mathrm{mM}$ Tris-HCl pH 7.5 containing Complete Protease Inhibitor Cocktail (Roche Diagnostics Corporation, Indianapolis, IN). After homogenization on ice 
in a glass Teflon homogenizer (20 strokes, household drill at full speed) the homogenate (total volume $1.5 \mathrm{ml}$ ) was centrifuged at $900 \mathrm{~g}$ for 10 minutes. The resulting supernatant was then centrifuged for 20 minutes at $10,000 \mathrm{~g}$. The resulting pellet was resuspended in the homogenization buffer and stored in aliquots for protein determination and Western blotting.

Immunoprecipitation. Twenty-four hours after isolation and plating on $10 \mathrm{~cm}$ plates, human hepatocytes were washed twice with ice-cold PBS. The hepatocytes

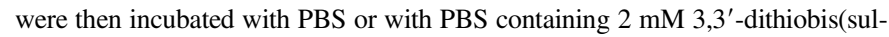
fosuccinimidyl proprionate) (DTSSP) for 2 hours at $4^{\circ} \mathrm{C}$. The crosslinking reaction was then stopped by incubating the hepatocytes with $20 \mathrm{mM}$ Tris- $\mathrm{HCl}$ for 15 minutes, followed by two washes with $5 \mathrm{ml}$ of ice-cold PBS per plate. One milliliter of ice-cold hypotonic homogenization solution containing $1 \mathrm{mM} \mathrm{NaCl}$, $5 \mathrm{mM}$ Tris-HCl pH 7.5, and Complete Protease Inhibitor Cocktail was added, and the hepatocytes were scraped from the plates. The hepatocytes were then homogenized using a household drill at full speed on ice for 20 strokes in a glass-Teflon homogenizer. The resulting homogenates were centrifuged in Eppendorf tubes at $900 \mathrm{~g}$ for 10 minutes. The resulting supernatants were centrifuged again at $10,000 \mathrm{~g}$ for 20 minutes, and the pellets containing a membrane enriched fraction were solubilized with PBS containing $0.1 \%$ NP40 for 10 minutes on ice. After centrifugation at $14,000 \mathrm{~g}$ for 30 minutes to remove insoluble particles, $1 \mu \mathrm{l}$ of OATP1B3 antibody (400B3) was added into each sample and incubated overnight at $4^{\circ} \mathrm{C}$ with end-over-end rotation. Twenty microliters of Dynabeads Protein G (Invitrogen) were washed twice with PBS containing $0.1 \% \mathrm{NP}-40$ before they were incubated with the antibody-containing samples for 24 hours at $4^{\circ} \mathrm{C}$. The magnetic beads were washed twice with $1 \mathrm{ml}$ PBS containing $0.1 \% \mathrm{NP}-40$ for 15 minutes, and the immunoprecipitated proteins were eluted with $200 \mu 11 \% \mathrm{TX}-100$ in $0.1 \mathrm{M}$ Glycine ( $\mathrm{pH}=2.5)$. After separation using SDS-PAGE and transfer to polyvinylidene fluoride membranes, coimmunoprecipitated OCT1 was detected with an anti-OCT1 antibody.

Proximity Ligation Assay. HEK293 cells were plated on poly-D-lysine pretreated eight-well chamber slides at 40,000 cells per well. Cells were transfected 24 hours later with His-tagged OATP1B 3 and OCT1 or with FLAG-tagged OATP1B3 and His-tagged ASBT plasmids at a 1:1 ratio. Fortyeight hours after transfection, cells were washed three times with PBS and fixed with PBS containing 2\% paraformaldehyde and $1 \%$ TX-100 for 10 minutes. After four washes with PBS, cells were blocked with 5\% normal donkey serum for 1 hour at room temperature and then incubated overnight at $4{ }^{\circ} \mathrm{C}$ with a combination of polyclonal anti-His and monoclonal OCT1 or polyclonal antiHis and monoclonal anti-FLAG primary antibodies in PBS containing 1\% normal donkey serum. The next day, slides were washed four times and then incubated with the Duolink In Situ PLA Probe Anti-Mouse PLUS in combination with the Duolink In Situ PLA Probe Anti-Rabbit MINUS (Sigma-Aldrich) at room temperatures for 1 hour. After two washes for 5 minutes each with Wash Buffer A (Sigma), ligation (at $37^{\circ} \mathrm{C}$ for 30 minutes) and amplification (at $37^{\circ} \mathrm{C}$ for 100 minutes) were performed following the manufacturer's protocols. Before mounting with Duolink In Situ Mounting Medium containing 4',6-diamidino-2phenylindole (DAPI; Sigma-Aldrich), cells were washed twice for 10 minutes each with Wash Buffer B and once for 1 minute with $0.01 \times$ Wash Buffer B.

Surface Biotinylation. HEK293 cells were plated at 600,000 cells per well on poly-D-lysine pretreated six-well plates. The next day the medium was changed, and different plasmid combinations were used for transfection. Twenty-four hours later for conditions including OCT1 or 48 hours later for conditions including
OATP1B1 or NTCP, cells were washed with ice-cold PBS and then treated with $1 \mathrm{mg} / \mathrm{ml}$ EZ-Link Sulfo-NHS-SS-Biotin (Thermo Fisher) in PBS for 2 hours at $4^{\circ}$ C. The reaction was stopped by washing with PBS containing $100 \mathrm{mM}$ Tris $(\mathrm{pH}=$ 7.5) for 15 minutes, and then the cells were lysed and harvested in Lysis Buffer (150 mM NaCl, 1 mM EDTA, 0.1\% SDS, $1 \%$ Triton X-100, $10 \mathrm{mM}$ Tris-HCl, pH $=7.5$ ) containing Complete Protease Inhibitor Cocktail for 10 minutes. Lysates were centrifuged at $10,000 \mathrm{~g}$ for 5 minutes, and the resulting supernatants were added to Streptavidin-coupled Dynabeads (Thermo Fisher) and rotated endover-end for 2 hours at room temperature. The beads were then centrifuged at $850 \mathrm{~g}$ for 2 minutes and washed twice for 5 minutes each with Lysis Buffer. Cell surface proteins were recovered by an incubation with Elution Buffer $(2 \times$ Laemmli buffer containing $10 \% \beta$-mercaptoethanol diluted with an equal volume of Lysis Buffer containing $2 \times$ Protease Inhibitors) for 30 minutes at room temperature followed by centrifugation at $850 \mathrm{~g}$ for 2 minute. Cell membrane proteins were heated at $50^{\circ} \mathrm{C}$ for 10 minutes and then separated using SDS-PAGE followed by immunoblotting as described (Zhang et al., 2017). The bands were quantified using Image Studio Lite Quantification Software (LI-COR, Lincoln, NE), and intensities of transporter proteins were normalized to the expression of $\mathrm{Na}^{+} / \mathrm{K}^{+}$ATPase. Transporter-mediated uptake was calculated in picomoles per milligram of total protein per minute. This number was then normalized to OATP1B3 surface expression levels. The surface expression of OATP1B3/EV was set to 1 (in Fig. 6 it was normalized to either OCT1/EV or OATP1B1/EV or NTCP/EV) and given as picomoles per normalized milligram per minute as follows: picomoles/(milligram of total protein $\times$ normalization ratio)/minute, where normalization ratio is the surface expression level $\mathrm{OATP1B}_{3} /$ surface expression level $_{\mathrm{Na}+\mathrm{K}+\text { ATPase. The apparent }}$ turnover number, $k_{\text {cat }}$, was calculated by normalizing $V_{\max }$ to the relative OATP1B3 surface expression levels.

Calculations and Statistics. All calculations were performed using Prism 8 (GraphPad Software Inc., San Diego, CA). Kinetic parameters were determined within the initial linear period of uptake after correction for protein and subtraction of uptake by the control cell line. Statistical analysis was performed with GraphPad Prism 8; $P<0.05$ was considered significant.

\section{Results}

OATP1B3 and OCT1 Interact in Human Hepatocytes. To investigate whether OATP1B3 would interact with other human liver proteins, Dualsystems AG performed a DUALmembrane screening with full-length OATP1B3 as the bait. Screening a human adult liver cDNA library resulted in the isolation of 51 cDNAs that were further analyzed and classified. Nineteen of these cDNAs were very likely unspecific interactors because they were found in $20-50 \%$ of the screenings, independent of the bait. Among the other 32 hits that were considered to be screen-specific interactors, OCT1, encoded by $S L C 22 A 1$, was found three times and was the only transporter. Additional hits were the human retinol-binding protein (Uniport identifier: Q5VY30) also found three times; the human cDNA FLJ54783 (B4DLG6) found twice; the chromosome 2 open reading frame 28 (D6W551) found twice; the Hepcidin antimicrobial peptide (Q6KC15) found four times; and a protein similar to the Macaca mulatta nuclear pore membrane
A

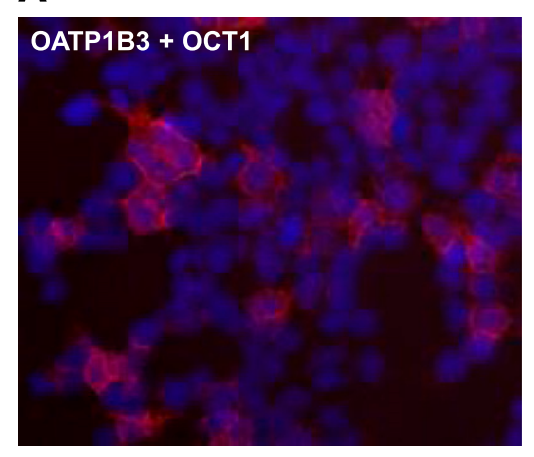

B

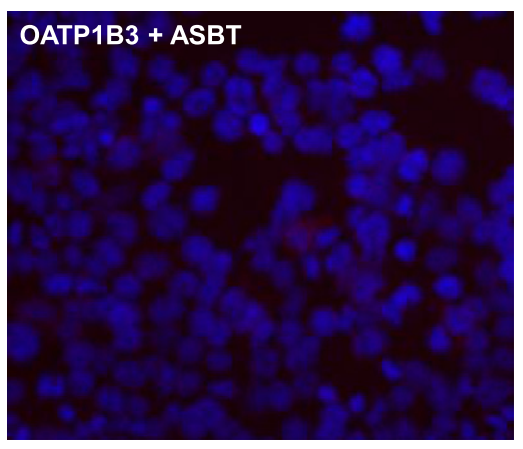

Fig. 2. OATP1B3 and OCT1 interact in transiently transfected HEK293 cells. Interaction of OATP1B3 with OCT1 is demonstrated using the proximity ligation assay. HEK293 cells were transfected with (A) OATP1B3-His and OCT1 plasmids (1:1), or (B) OATP1B3-FLAG and ASBT-His plasmids (1:1). Twenty-four hours after transfection HEK293 cells were fixed and incubated with a combination of corresponding antibodies. After incubation with the respective secondary antibodies and the ligation reaction mix, interactions can be detected in red. The blue color represents DAPI staining of the nuclei. 
A

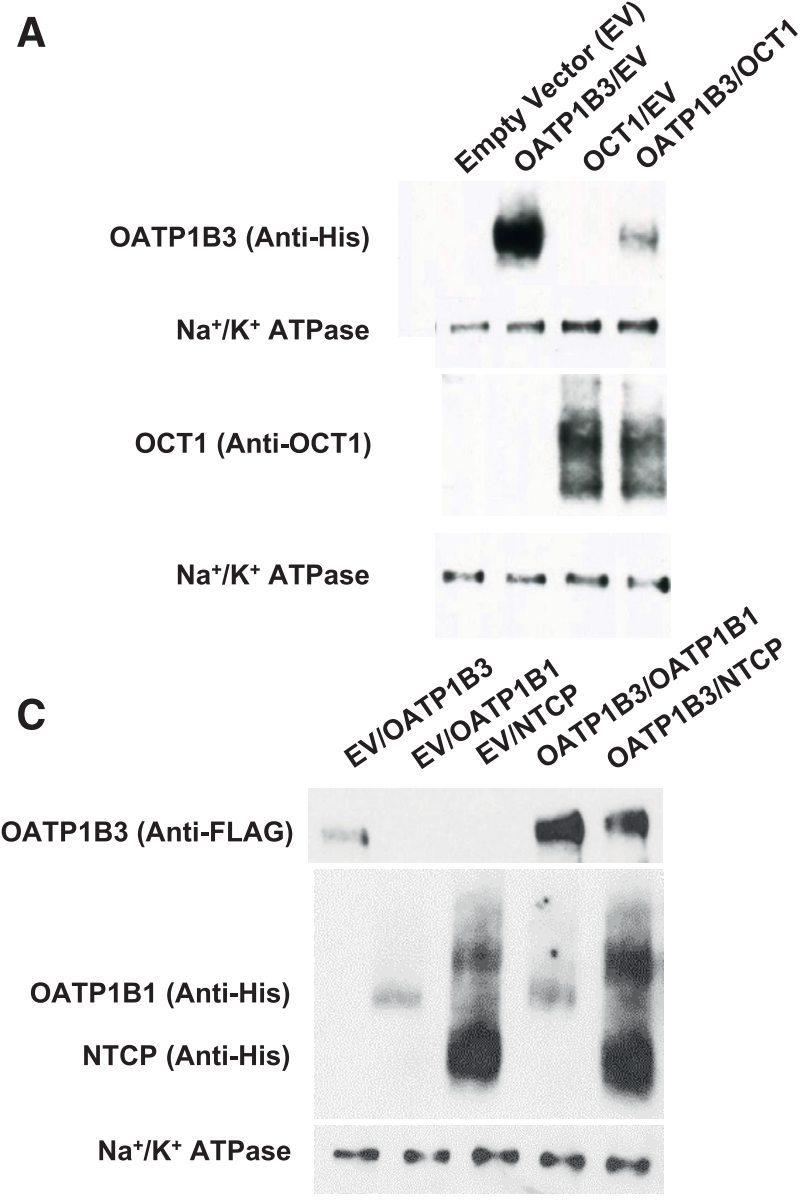

B

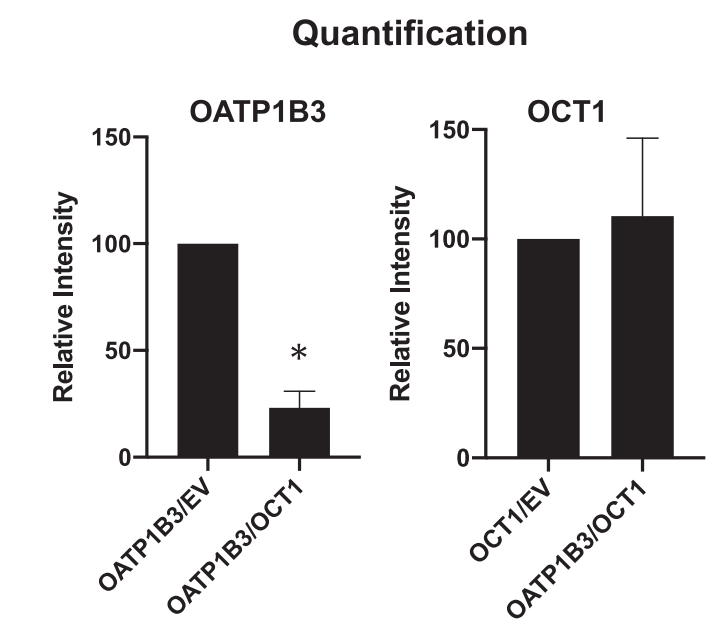

D

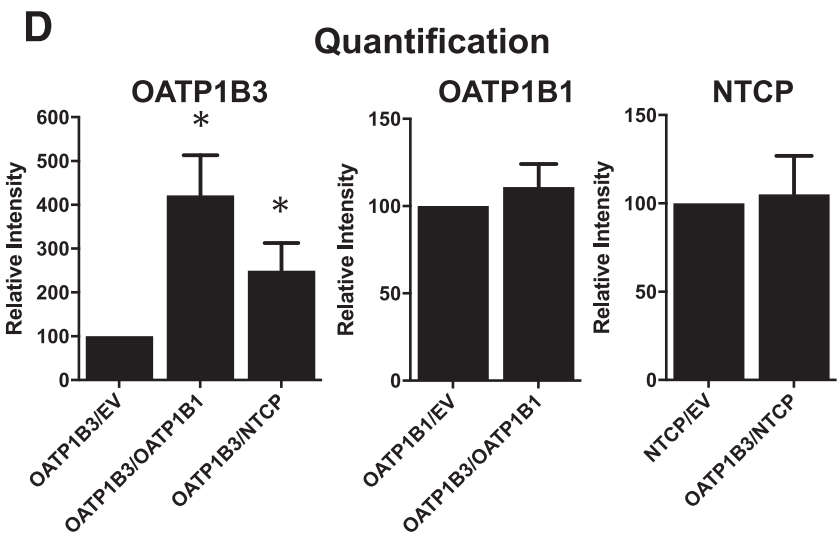

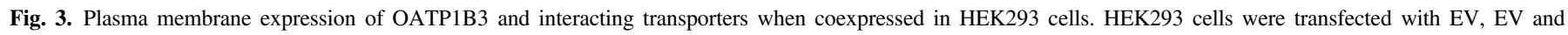

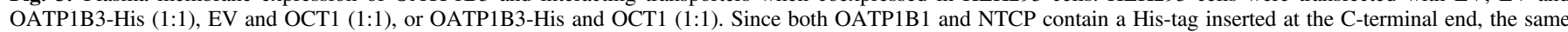

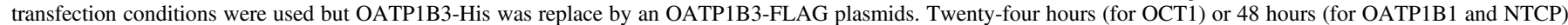

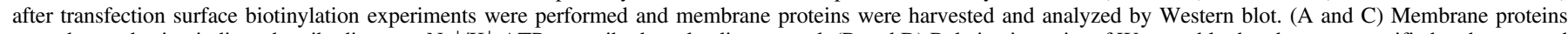

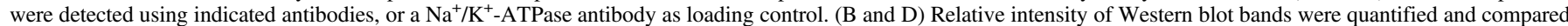

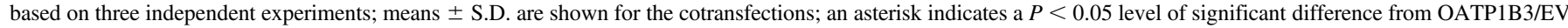
by one-tailed $t$ test.

glycoprotein 210 (H9F470) found twice. In addition, there were 16 single hits of proteins that could be true interactors but also represent common false positives in such screens. Given that OCT1, like OATP1B3, is a drug uptake transporter expressed at the basolateral membrane of human hepatocytes, we further investigated this potential interaction by performing coimmunoprecipitation experiments. As can be seen in Fig. 1A, OCT1 could be detected with an OCT1-specific antibody on a Western blot of proteins that were immunoprecipitated from solubilized human hepatocytes with the 400B3 anti-OATP1B3 antibody. The signal for OCT1 at approximately $75 \mathrm{kDa}$ was increased in the presence of the crosslinker DTSSP. To further confirm the interaction of OATP1B3-OCT1 in human liver, we performed proximity ligation assays on frozen sections of human livers. The proximity ligation assay detects interacting proteins that are less than $40 \mathrm{~nm}$ apart using two antibodies raised in different species (Söderberg et al., 2006). If these two proteins are less than $40 \mathrm{~nm}$ apart, the assay will result in a red signal. We previously used this assay to demonstrate OATP1B3NTCP interactions in frozen human liver slices (Zhang et al., 2017). When we incubated frozen human liver sections only with the monoclonal antibody recognizing OCT1, no red signal was generated (Fig. 1B; OCT1 only). However, when the monoclonal anti-OCT1 antibody was used together with the polyclonal anti-OATP1B3 antibody
(K28), interactions between the two transporters were evident by the red signals (Fig. 1B; OATP1B3 + OCT1).

OATP1B3 and OCT1 Interact in HEK293 Cells. To characterize the functional consequences of the OATP1B3-OCT1 interactions we wanted to use transiently transfected HEK293 cells. Because HEK293 cells did not survive 48 hours after transient transfection with the OCT1 plasmid, we determined protein expression and function at 24 hours whenever OCT1 was cotransfected. Using the proximity ligation assay we first tested whether these protein-protein interactions also occur when both transporters were expressed in HEK293 cells. The results in Fig. 2A confirmed that OATP1B3 and OCT1 do interact when expressed in HEK293 cells. As a negative control we transfected OATP1B3 with ASBT, a transporter that is expressed in cholangiocytes and enterocytes but not in hepatocytes. The lack of a signal (Fig. 2B) clearly demonstrates that the interaction of OATP1B3 with OCT1 in HEK293 cells is not due to unspecific overexpression of any membrane protein but is due to specific interactions between these two transport proteins.

Plasma Membrane Expression of OATP1B3 Is Affected by Hetero-Oligomerization in HEK293 Cells. After demonstrating the interaction between OATP1B3 and OCT1 in HEK293 cells, we asked whether expression at the plasma membrane would be affected by coexpression of OATP1B3 with OCT1. In addition, since we recently 
A
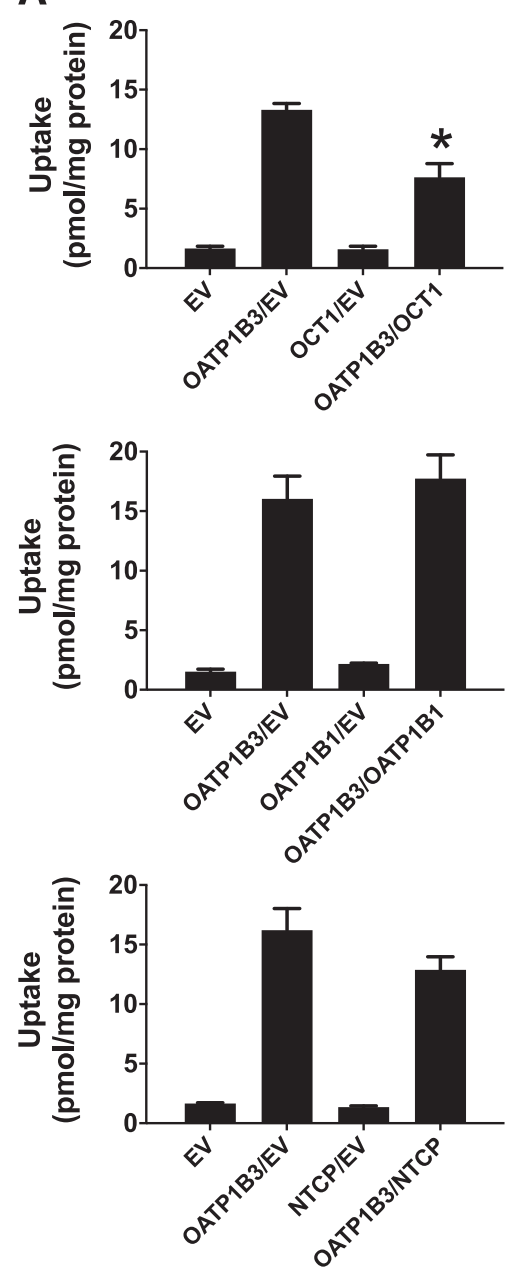

B
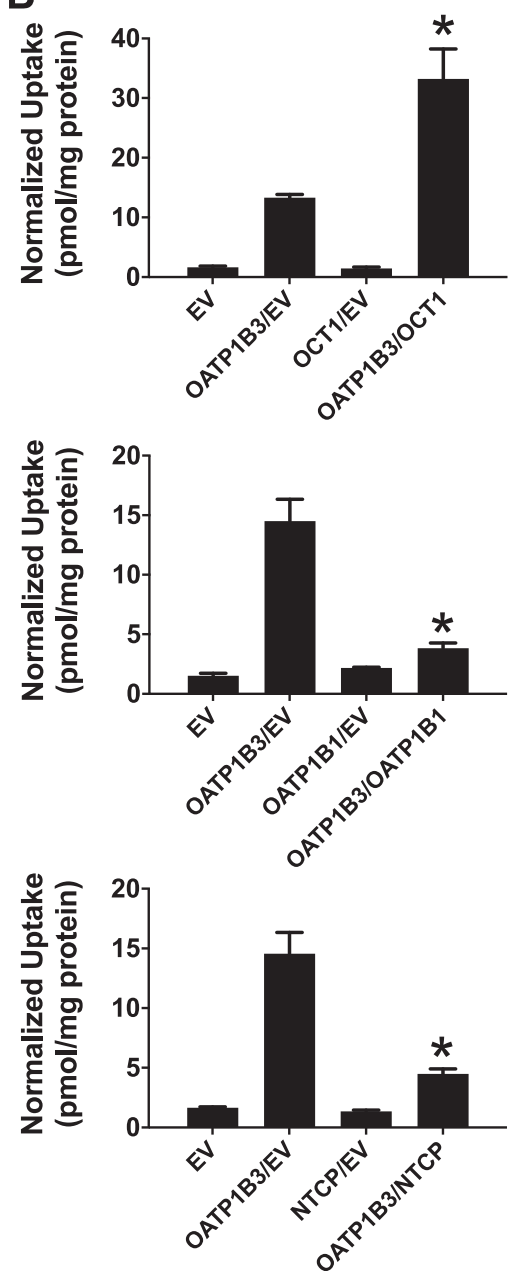

Fig. 4. Functional consequences for OATP1B 3 when coexpressed with OCT1, OATP1B1 or NTCP. Uptake experiments with $1 \mu \mathrm{M}$ ${ }^{3} \mathrm{H}-\mathrm{CCK}-8$ were performed 24 hours (OCT1) or 48 hours (OATP1B1 and NTCP) after HEK293 cells were transiently transfected with an equivalent amount cDNA with the indicated combination of plasmids or EV. Uptake was terminated after 1 minute and normalized by total protein concentration (A) or surface expressed protein (after normalizing for expression of $\mathrm{Na}^{+} / \mathrm{K}^{+}$ ATPase) (B). The means \pm S.D. of three experiments $(n=3)$ are shown; an asterisk indicates a $P<0.05$ level of significant difference from OATP1B3/EV by one-tailed $t$ test. determined that OATP1B3 can also interact with OATP1B1 and NTCP (Zhang et al., 2017), we also investigated the effect of coexpression of OATP1B3 with these two transporters. OATP1B3 was transfected in HEK293 cells either alone (cotransfected with empty vector) or together with either OCT1, OATP1B1, or NTCP. After 24 hours for OCT1 or 48 hours for OATP1B1 and NTCP, membrane proteins were labeled and isolated using the biotin-streptavidin system. Plasma membrane expression of OATP1B3, OCT1, OATP1B1, and NTCP was then quantified by SDS-PAGE and Western blot. As can be seen in Fig. 3A, no OATP1B3 was visible when only empty vector or OCT1 was transfected. When OATP1B3 was transfected together with empty vector a clear signal was seen that was reduced when OATP1B3 was coexpressed with OCT1. After quantification and normalization for the $\mathrm{Na}^{+} / \mathrm{K}^{+}$ATPase signal, we calculated that coexpression of OCT1 reduced the OATP1B 3 signal to about 20\% (Fig. 3B). In contrast, expression levels for OCT1 cotransfected with OATP1B3 did not result in any significant changes in surface expression as compared with cotransfection with empty vector (Fig. 3, A and B). Interestingly, OATP1B3 protein expression was increased significantly when coexpressed with either OATP1B1 or NTCP (Fig. 3C). Quantification and normalization with the $\mathrm{Na}^{+} / \mathrm{K}^{+}$ ATPase signal revealed about a 4.5-fold and 2.5-fold increase in plasma membrane expression when coexpressed with OATP1B1 and NTCP, respectively, whereas expression of OATP1B1 and NTCP was not affected (Fig. 3D). Given that only transporters expressed at the surface of HEK293 cells can transport their substrates, these surface expression levels have to be taken into consideration for functional studies.
Hetero-Oligomerization in HEK293 Cells Affects OATP1B3-Mediated CCK-8 Transport. We next determined whether the interaction between OATP1B3 and one of the other interacting proteins (OCT1, OATP1B1, or NTCP) also affects OATP1B3 transport function. We compared uptake of the OATP1B3 specific substrate, CCK-8, into HEK293 cells transfected with OATP1B3 and empty vector, or OATP1B3 and the respective interacting transporter. Twenty-four hours (for cotransfection with OCT1) or 48 hours (for cotransfection with OATP1B1 or NTCP) after transfection, uptake of $1 \mu \mathrm{M}{ }^{3} \mathrm{H}-\mathrm{CCK}-8$ was measured for 1 minute at $37^{\circ} \mathrm{C}$. As can be seen in Fig. $4 \mathrm{~A}$, uptake of CCK-8 was detected in OATP1B3 expressing HEK293 cells but was absent in cells transfected with empty vector, OCT1, OATP1B1, or NTCP. When OATP1B3 was coexpressed with OCT1, uptake was reduced to $50 \%$ (Fig. 4A, top). However, when OATP1B3 was coexpressed with either OATP1B1 or NTCP, uptake of CCK-8 by OATP1B3 was not significantly affected (Fig. 4A, middle and bottom). These results suggest that uptake of CCK-8 in human hepatocytes could be affected by the expression level of OCT1 and should be increased if expression of OCT1 was decreased. However, expression levels of OATP1B1 or NTCP should not affect CCK-8 transport. When uptake of CCK-8 was normalized for plasma membrane expressed OATP1B3, transport was increased 2.7-fold (Fig. 4B, top). Interestingly, an opposite effect was observed with OATP1B1 or NTCP. After normalization for membrane expressed OATP1B3, uptake of CCK8 was reduced dramatically (Fig. 4B, middle and bottom). We next determined the initial linear portion of CCK- 8 uptake for OATP1B3 expressed alone and coexpressed with the three different transporters to 

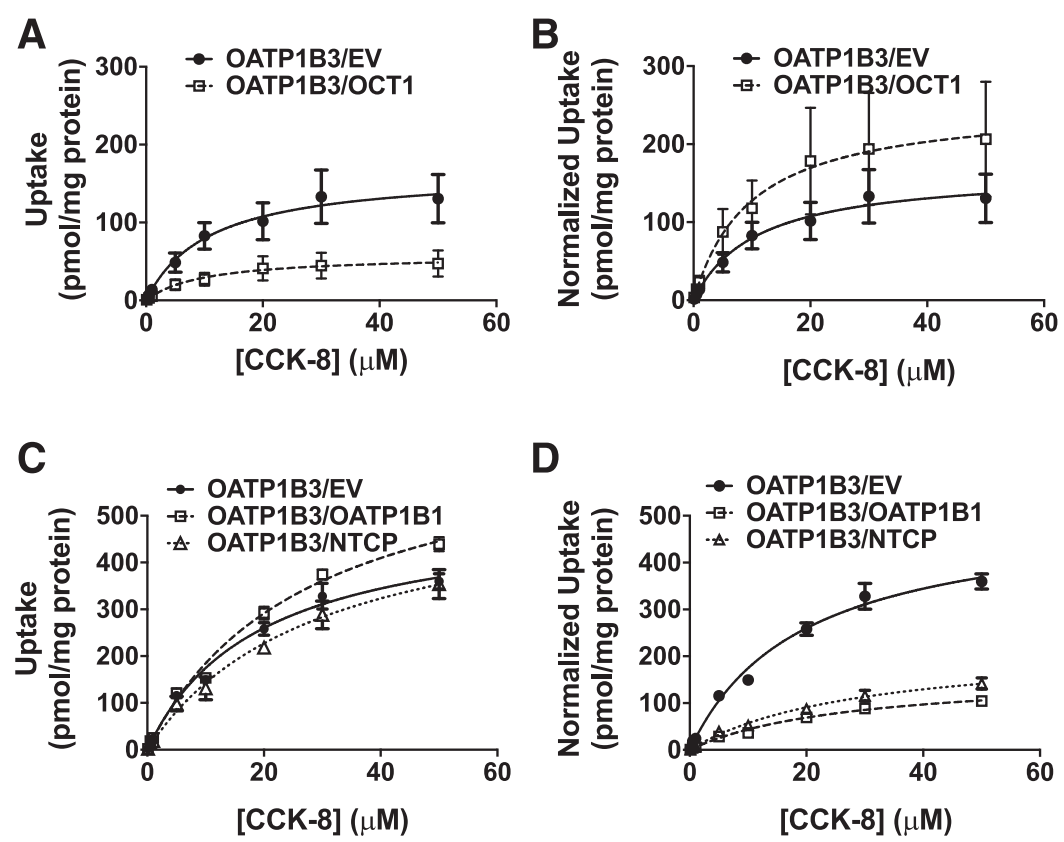

Fig. 5. Effects of coexpression of interacting transporters on the kinetics of OATP1B3-mediated CCK-8 uptake. Uptake of increasing concentrations of ${ }^{3} \mathrm{H}-\mathrm{CCK}-8(0.1-50 \mu \mathrm{M})$ was measured at $37^{\circ} \mathrm{C}$ for 1 minute in HEK293 cells transiently transfected with $500 \mathrm{ng}$ of empty vector, or $250 \mathrm{ng}$ of empty vector mixed with $250 \mathrm{ng}$ of OATP1B3 (OATP1B3/EV), or $250 \mathrm{ng}$ of OATP1B3 mixed with $250 \mathrm{ng}$ of respective interacting transporters. Uptake was normalized by total protein (A and $\mathrm{C}$ ) and plasma membrane expressed OATP1B3 (B and D). After subtracting the values obtained with empty vector transfected cells, net OATP1B3mediated uptake was fitted to the Michaelis-Menten equation to obtain $K_{\mathrm{m}}$ and $V_{\max }$ values. Means \pm S.E. of seven ( $(\mathrm{A}$ and B) for interaction with OCT1) or three $((\mathrm{C}$ and $\mathrm{D})$ for interaction with either OATP1B1 or NTCP) individual experiments each performed with triplicate determinations are given. Circles and a solid line represent OATP1B3/EV transfected cells, whereas squares and a dashed line represent OATP1B3/interacting transporter transfected cells. be able to characterize kinetics. Based on these experiments, we performed CCK-8 uptake experiments with increasing CCK-8 concentrations $(0.1-50 \mu \mathrm{M})$ at 1 minute. As shown in Fig. 5, A and B (summarized in Table 1), OATP1B3-mediated CCK-8 uptake was measured 24 hours after transfection and was saturable with a $K_{\mathrm{m}}$ value of $10.9 \pm 2.4 \mu \mathrm{M}$ and a $V_{\max }$ value of $166 \pm 12.4 \mathrm{pmol} / \mathrm{mg}$ protein $\times$ $\min$. When OCT1 was coexpressed with OATP1B3, the $K_{\mathrm{m}}$ value stayed the same, but the $V_{\max }$ decreased to $58.1 \pm 6.0$. When the values were normalized for the plasma membrane expression of OATP1B3, the $K_{\mathrm{m}}$ value did not change, but the $V_{\max }$ increased to $253 \pm 26.2 \mathrm{pmol} / \mathrm{mg} \times$ min. Forty-eight hours after cotransfection with either OATP1B1 or NTCP, the $K_{\mathrm{m}}$ values tended to be increased compared with the 24-hour values but were not affected by coexpression of OATP1B1 or NTCP (Table 2). However, the normalized $V_{\max }$ values decreased significantly when compared with the cotransfection of OATP1B3 with empty vector (Fig. 5; Table 2).

Hetero-Oligomerization in HEK293 Cells Does Not Seem to Affect the Function of OCT1, OATP1B1, and NTCP. To investigate whether the function of the OATP1B3 partner proteins would be affected by the coexpression, we measured uptake of representative model substrates for OCT1 (metformin), OATP1B1 (low concentration of estrone-3-sulfate), and NTCP (taurocholate) after cotransfection in HEK293 cells. The results in Fig. 6 demonstrate that the function of the three interacting transport proteins was not affected by coexpression with OATP1B3.

Knockdown of OCT1 in Human Hepatocytes Stimulates OATP1B3-Mediated CCK-8 Uptake. If these findings are important for normal physiology/pharmacology they have to be confirmed in a more relevant system. The decreased uptake of OATP1B3-mediated
CCK-8 in the presence of OCT1 (Fig. 4A) led us to hypothesize that if OCT1 expression in human hepatocytes were reduced, this would lead to an increased OATP1B3-mediated CCK- 8 uptake. To test our hypothesis, we used adenoviral vectors that contained either a nontarget or scrambled shRNA as control, or OCT1 shRNA. We plated human hepatocytes, treated them with the respective adenoviral vectors and cultured them for 11 days. We used one batch of cryopreserved human hepatocytes (HUM4180A) and two different batches of freshly isolated human hepatocytes (KHH134 and KHH135). On day 11, we measured uptake of CCK-8 in the absence or presence of $100 \mu \mathrm{M}$ bromosulfophthalein, a known substrate and inhibitor of OATP1B3. In addition, we isolated a membrane enriched fraction for Western blotting. As can be seen in Fig. 7A, the protein expression of OCT1 was reduced in the cryopreserved hepatocytes for the fully glycosylated protein and in the freshly isolated hepatocytes mainly for the deglycosylated protein. As a consequence, CCK-8 uptake increased in each batch of hepatocytes that was treated with the OCT1 shRNA as compared with the scrambled shRNA, confirming our hypothesis.

\section{Discussion}

In the present study we used coimmunoprecipitation and proximity ligation assays to demonstrate that OATP1B3 and OCT1 interact with each other when expressed in HEK293 cells as well as in human hepatocytes. Furthermore, coexpression with either OCT1, OATP1B1, or NTCP affects the expression levels of OATP1B3 and its function. These results demonstrate two important findings: 1) coexpression of OATP1B3 with OCT1 affects the membrane expression of OATP1B3, and 2) this coexpression directly affects the function of OATP1B3. The

TABLE 1

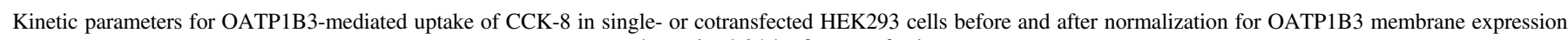
determined $24 \mathrm{~h}$ after transfection

\begin{tabular}{|c|c|c|c|}
\hline Transfection condition & $\begin{array}{c}K_{\mathrm{m}} \\
\mu \mathrm{M}(95 \% \mathrm{CI})\end{array}$ & $\begin{array}{c}V_{\max } \\
\mathrm{pmol} / \mathrm{mg} \text { protein } \times \min (95 \% \mathrm{CI})\end{array}$ & $\begin{array}{c}\text { Normalized } V_{\max } \\
\mathrm{pmol} / \mathrm{mg} \text { protein } \times \min (95 \% \mathrm{CI})\end{array}$ \\
\hline OATP1B3/EV & $10.9 \pm 2.4(6.1-15.6)$ & $166 \pm 12.4(141-191)$ & $166 \pm 12.4(141-191)$ \\
\hline OATP1B3/OCT1 & $9.8 \pm 3.1(3.6-16.0)$ & $58.1 \pm 6.0(46.1-70.2)$ & $253 \pm 26.2(200-305)$ \\
\hline
\end{tabular}

CI, confidence interval. 
TABLE 2

Kinetic parameters for OATP1B3-mediated uptake of CCK-8 in single- or cotransfected HEK293 cells before and after normalization for OATP1B3 membrane expression determined $48 \mathrm{~h}$ after transfection

\begin{tabular}{|c|c|c|c|}
\hline Transfection condition & $\begin{array}{c}K_{\mathrm{m}} \\
\mu \mathrm{M}(95 \% \mathrm{CI})\end{array}$ & $\begin{array}{c}V_{\max } \\
\mathrm{pmol} / \mathrm{mg} \text { protein } \times \min (95 \% \mathrm{CI})\end{array}$ & $\begin{array}{c}\text { Normalized } V_{\max } \\
\mathrm{pmol} / \mathrm{mg} \text { protein } \times \min (95 \% \mathrm{CI})\end{array}$ \\
\hline OATP1B3/EV & $19.2 \pm 3.3(12.3-26.1)$ & $510 \pm 36.7(433-586)$ & $510 \pm 36.7(433-586)$ \\
\hline OATP1B3/OATP1B1 & $27.4 \pm 3.6(19.9-34.9)$ & $690 \pm 43.7(599-781)$ & $164 \pm 10.3(142-185)$ \\
\hline OATP1B3/NTCP & $29.2 \pm 7.2(14.2-44.2)$ & $559 \pm 67.9(418-701)$ & $224 \pm 27.2(167-281)$ \\
\hline
\end{tabular}

CI, confidence interval.

coexpression of OATP1B3 with OCT1 leads to decreased uptake prior to normalization. Therefore, when knocking OCT1 expression down in human hepatocytes we expected to see increased uptake. Indeed, increased uptake for CCK-8 and for sodium-independent uptake of taurocholate was observed (Fig. 7). These results suggest that changes at the protein level of OCT1, either due to disease or due to polymorphisms, will affect the disposition of drugs that are transported mainly by OATP1B3, such as, e.g., telmisartan (Ishiguro et al., 2006). Preliminary data from experiments with HEK293 cells (data not shown) suggest that OCT1 affects the expression of OATP1B1 and NTCP in a similar way. Thus, OCT1 expression levels would affect not only the disposition of OCT1 substrates but also that of drugs transported by OATPs and NTCP. Regarding the function, coexpression of OCT1 increased the $V_{\max }$ value of OATP1B3-mediated CCK-8 uptake after normalization for plasma membrane expression. These experiments had to be performed 24 hours after transfection because OCT1 overexpression damaged the HEK293 cells when we let expression go for 48 hours. In contrast, coexpression experiments with either OATP1B 1 or NTCP could be performed at 48 hours without any cell viability issues. In the presence of OATP1B 1 or NTCP, $V_{\max }$ values of OATP1B3-mediated CCK-8 uptake were decreased as compared with when OATP1B3 was expressed alone after normalization for plasma membrane expression. Given that after normalization the values are corrected for the number of OTP1B3 transporters at the plasma membrane, this clearly means that the increase in the presence of OCT1 is due to an increase in turnover number. However, the decrease in the presence of either OATP1B1 or NTCP demonstrated that the turnover number decreased. These changes of an increased or decreased apparent turnover number $\left(k_{\text {cat }}\right)$ for OATP1B3 suggest that OATP1B3 function is also affected in vivo in human hepatocytes by coexpression of other proteins, in particular other transport proteins. Thus, besides the well known transcriptional and posttranslational regulation of expression and function, these drug transporters seem to be regulated at an additional level involving protein-protein interactions.

Under normal physiologic conditions OATP1B3 is exclusively expressed at the basolateral membrane of human hepatocytes with the highest expression around the central vein (König et al., 2000; Cui et al., 2003). A similar lobular expression was also reported for mouse OCT1 (Chen et al., 2014). In mice, the corresponding proteins OCT1 and OATP1B2, respectively, have been shown to have differential regulation in a mouse model of nonalcoholic steatohepatitis due to a methionineand choline-deficient diet. In these animals, OATP1B2 is downregulated (Clarke et al., 2014), whereas OCT1 expression goes up compared with healthy controls (Clarke et al., 2015). Interestingly, OATP1B3 protein expression was much lower in nonalcoholic steatohepatitis livers when compared with normal livers (Clarke et al., 2014), but there is no information available yet on OCT1 expression under the same conditions. Although we do not yet know the mechanisms by which the OCT1-OATP1B3 protein interaction results in downregulated OATP1B3 protein, a similar protein-protein interaction between these corresponding mouse proteins could be responsible for the observed differential regulation.

Unlike OCT1, OATP1B1 and NTCP have an even distribution throughout the liver lobule (Stieger et al., 1994; König et al., 2000), which could potentially explain the reversed effect on OATP1B3 compared with OCT1.

We have demonstrated that coexpression of OATP1B3 with other transporters can affect its function. Thus, because in human hepatocytes both transporters are expressed in the same hepatocyte, this coexpression likely affects the physiologic function of OATP1B3 and/or its ability to clear drugs. When characterizing and predicting drug-drug interactions researchers normally use HEK293 or Chinese hamster ovary cells that express a single transporter (Hirano et al., 2006; Noé et al., 2007; Gui et al., 2008). However, given that these drug transporters might be affected by coexpressed transporters, these single expression systems do
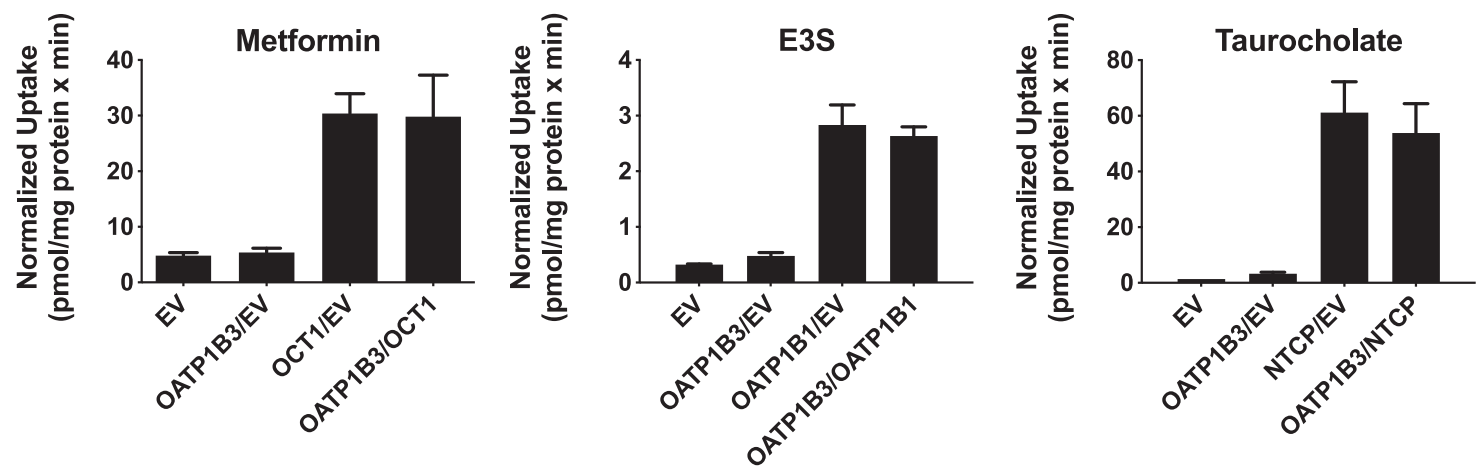

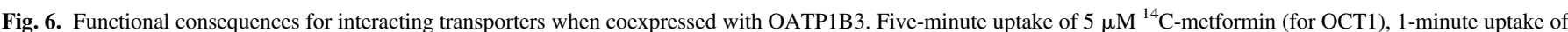

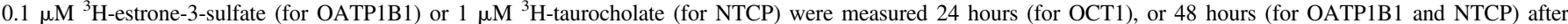

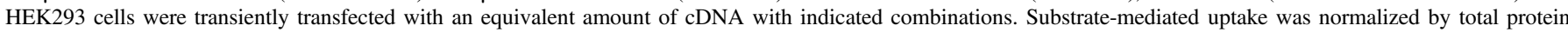

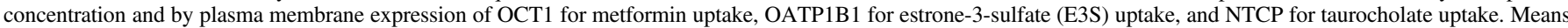
\pm S.D. are given for three experiments $(n=3)$ each performed in triplicates. 
HUM4180A
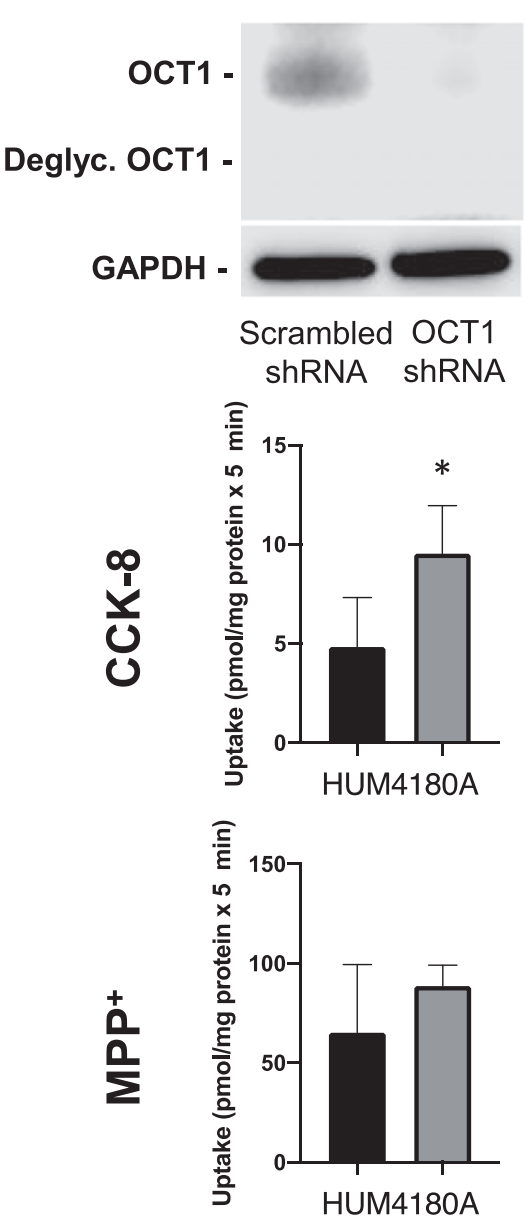

KHH134
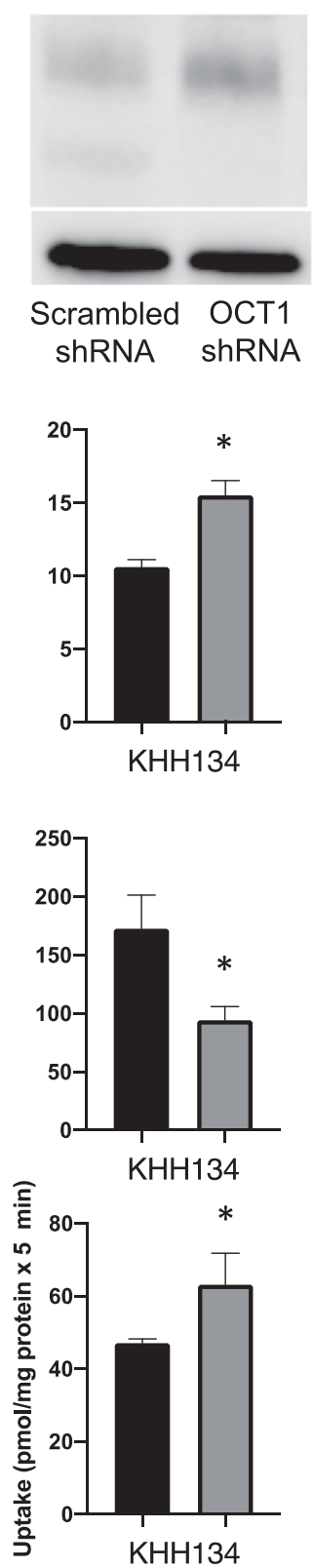

KHH135
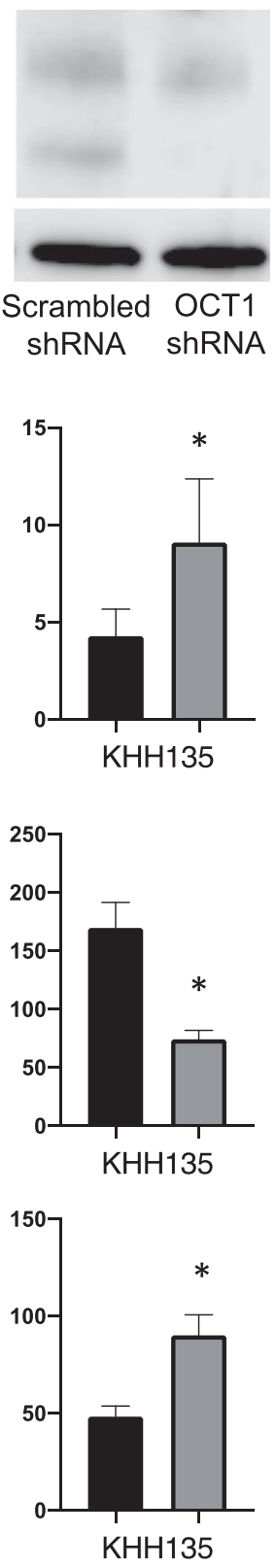

Fig. 7. Effect of knocking down OCT1 in primary cultured human hepatocytes on OATP1B3mediated uptake. Western blots of protein isolated from additional wells were probed with a monoclonal OCT1 antibody and a polyclonal glyceraldehyde-3-phosphate dehydrogenase (GAPDH) antibody as a loading control. Five-minute uptake of $1 \mu \mathrm{M}{ }^{3} \mathrm{H}-\mathrm{CCK}-8,5 \mathrm{nM}{ }^{3} \mathrm{H}-\mathrm{MPP}^{+}$, and $1 \mu \mathrm{M}$ ${ }^{3} \mathrm{H}$-taurocholate (TCA; in sodium-free medium) was measured 11 days after treatment of plated human hepatocytes with adenoviral scrambled (black bars) or OCT1-shRNA (gray bars) on 12well plates. Means \pm S.D. of triplicate determinations are shown for three independent hepatocyte preparations; an asterisk indicates a $P<0.05$ level of significant difference by one-tailed $t$ test. not really reflect the situation in human hepatocytes, and results obtained in the single transfection systems could be misleading. In the future, single transporter experiments with several drugs, e.g., statins or sartans, should be evaluated to ensure they are equivalent to multiple transporter expression systems. If not, multiple transporter expression systems or a combination of single and multiple transporter systems should be used for pharmacological and toxicological studies.

Regulation of OATP1B3 and other OATPs has mainly been studied at the transcriptional level [reviewed in Hagenbuch and Stieger (2013)]. Limited information is available for posttranslational regulation via protein kinase C activation (Guo and Klaassen, 2001; Köck et al., 2010; Powell et al., 2014; Hong et al., 2015), and several rodent OATPs have been reported to interact with adapter proteins of the PDZ family [summarized in Stieger and Hagenbuch (2014)]. In particular, a recent study nicely demonstrated that rat OATP1A4 was only trafficked efficiently to the basolateral hepatocyte membrane if
OATP1A1, which contains a PDZ consensus motif, is also expressed (Wang et al., 2019). Furthermore, the authors speculated that there is a similar mechanism in human hepatocytes where the C-terminal amino acid sequence ETHC in OATP1B1 was predicted to bind to PDZK1. Our findings that OATP1B3 expression at the plasma membrane is increased when coexpressed with OATP1B1 could potentially be explained by this mechanism and will need to be tested experimentally. The interaction between coexpressed transporters suggests that they could also be coregulated at the posttranslational level. Thus, future experiments will determine whether this indeed is the case.

The interaction between carbonic anhydrase II and the epithelial sodium-proton exchanger (NHE3) results in increased activity of NHE3 (Krishnan et al., 2015). In the case of carbonic anhydrase and NHE3, functionally active carbonic anhydrase is required. Thus, in the future, we will need to test how the observed functional consequences are 
modified when transporters with inactivating or stimulating polymorphisms are coexpressed.

It still has to be determined exactly how these protein-protein interactions are mediated. We believe the different proteins may be integrated into lipid raft domains similar to what as has been shown for rat NTCP (Molina et al., 2008), rat OCTN2 (Czeredys et al., 2013) and rat OAT3 (Srimaroeng et al., 2013). Another possibility is that the interactions may involve PDZ motifs or "tetraspanin webs" like in the case of OCTs.

In summary, we have shown that the hepatocellular drug transporters OATP1B3 and OCT1 interact with each other and that this interaction can increase OATP1B3-mediated uptake clearance $\left(V_{\max } / K_{\mathrm{m}}\right)$ of CCK8 , but reduce the plasma membrane expression level of OATP1B3. The interaction between OATP1B3 and OATP1B1, or NTCP led to an opposite effect, decreasing the uptake clearance of OATP1B3 but increasing its plasma membrane expression level. These findings are important because they suggest that the clearance of endogenous compounds, as well as of drugs, can be affected by coexpressed proteins. Thus, the characterization of these drug transporters in single transfected recombinant systems might well lead to over- or underestimation of the effects of drug-drug interactions. Therefore, we should consider test systems that coexpress the involved transporters for such characterizations.

\section{Authorship Contributions}

Participated in research design: Ruggiero, Zhang, Hagenbuch.

Conducted experiments: Ruggiero, Zhang, Hagenbuch.

Performed data analysis: Ruggiero, Zhang, Hagenbuch.

Wrote or contributed to the writing of the manuscript: Ruggiero, Zhang, Hagenbuch.

\section{References}

Bednarczyk D and Boiselle C (2016) Organic anion transporting polypeptide (OATP)-mediated transport of coproporphyrins I and III. Xenobiotica 46:457-466.

Boxberger KH, Hagenbuch B, and Lampe JN (2014) Common drugs inhibit human organic cation transporter 1 (OCT1)-mediated neurotransmitter uptake. Drug Metab Dispos 42:990-995.

Chen L, Shu Y, Liang X, Chen EC, Yee SW, Zur AA, Li S, Xu L, Keshari KR, Lin MJ, et al (2014) OCT1 is a high-capacity thiamine transporter that regulates hepatic steatosis and is a target of metformin. Proc Natl Acad Sci USA 111:9983-9988.

Clarke JD, Dzierlenga AL, Nelson NR, Li H, Werts S, Goedken MJ, and Cherrington NJ (2015) Mechanism of altered metformin distribution in nonalcoholic steatohepatitis. Diabetes 64 3305-3313.

Clarke JD, Hardwick RN, Lake AD, Canet MJ, and Cherrington NJ (2014) Experimental nonalcoholic steatohepatitis increases exposure to simvastatin hydroxy acid by decreasing hepatic organic anion transporting polypeptide expression. J Pharmacol Exp Ther 348:452-458.

Claro da Silva T, Polli JE, and Swaan PW (2013) The solute carrier family 10 (SLC10): beyond bile acid transport. Mol Aspects Med 34:252-269.

Cui Y, König J, Nies AT, Pfannschmidt M, Hergt M, Franke WW, Alt W, Moll R, and Keppler D (2003) Detection of the human organic anion transporters SLC21A6 (OATP2) and SLC21A8 (OATP8) in liver and hepatocellular carcinoma. Lab Invest 83:527-538.

Czeredys M, Samluk Ł, Michalec K, Tu³odziecka K, Skowronek K, and Na ${ }^{3} \hat{c} c z$ KA (2013) Caveolin-1--a novel interacting partner of organic cation/carnitine transporter (Octn2): effect of protein kinase $\mathrm{C}$ on this interaction in rat astrocytes. PLoS One 8:e82105.

Gorboulev V, Ulzheimer JC, Akhoundova A, Ulzheimer-Teuber I, Karbach U, Quester S, Baumann C, Lang F, Busch AE, and Koepsell H (1997) Cloning and characterization of two human polyspecific organic cation transporters. DNA Cell Biol 16:871-881.

Gui C, Miao Y, Thompson L, Wahlgren B, Mock M, Stieger B, and Hagenbuch B (2008) Effect of pregnane $\mathrm{X}$ receptor ligands on transport mediated by human OATP1B1 and OATP1B3. Eur $J$ Pharmacol 584:57-65.

Guo GL and Klaassen CD (2001) Protein kinase C suppresses rat organic anion transporting polypeptide 1- and 2-mediated uptake. J Pharmacol Exp Ther 299:551-557.

Hagenbuch B and Stieger B (2013) The SLCO (former SLC21) superfamily of transporters. Mol Aspects Med 34:396-412.
Hirano M, Maeda K, Shitara Y, and Sugiyama Y (2006) Drug-drug interaction between pitavastatin and various drugs via OATP1B1. Drug Metab Dispos 34:1229-1236.

Hong M, Hong W, Ni C, Huang J, and Zhou C (2015) Protein kinase C affects the internalization and recycling of organic anion transporting polypeptide 1B1. Biochim Biophys Acta 1848: 2022-2030.

Ishiguro N, Maeda K, Kishimoto W, Saito A, Harada A, Ebner T, Roth W, Igarashi T, and Sugiyama Y (2006) Predominant contribution of OATP1B3 to the hepatic uptake of telmisartan, an angiotensin II receptor antagonist, in humans. Drug Metab Dispos 34:1109-1115. Ismair MG, Stieger B, Cattori V, Hagenbuch B, Fried M, Meier PJ, and Kullak-Ublick GA (2001) Hepatic uptake of cholecystokinin octapeptide by organic anion-transporting polypeptides OATP4 and OATP8 of rat and human liver. Gastroenterology 121:1185-1190.

Köck K, Koenen A, Giese B, Fraunholz M, May K, Siegmund W, Hammer E, Völker U, Jedlitschky G, Kroemer HK, et al. (2010) Rapid modulation of the organic anion transporting polypeptide 2B1 (OATP2B1, SLCO2B1) function by protein kinase C-mediated internalization. J Biol Chem 285:11336-11347.

Koepsell H (2013) The SLC22 family with transporters of organic cations, anions and zwitterions. Mol Aspects Med 34:413-435.

Koepsell H, Lips K, and Volk C (2007) Polyspecific organic cation transporters: structure, function, physiological roles, and biopharmaceutical implications. Pharm Res 24:1227-1251.

König J (2011) Uptake transporters of the human OATP family: molecular characteristics, substrates, their role in drug-drug interactions, and functional consequences of polymorphisms. Handb Exp Pharmacol (201):1-28.

König J, Cui Y, Nies AT, and Keppler D (2000) Localization and genomic organization of a new hepatocellular organic anion transporting polypeptide. J Biol Chem 275:23161-23168.

Krishnan D, Liu L, Wiebe SA, Casey JR, Cordat E, and Alexander RT (2015) Carbonic anhydrase II binds to and increases the activity of the epithelial sodium-proton exchanger, NHE3. Am J Physiol Renal Physiol 309:F383-F392.

Molina H, Azocar L, Ananthanarayanan M, Arrese M, and Miquel JF (2008) Localization of the sodium-taurocholate cotransporting polypeptide in membrane rafts and modulation of its activity by cholesterol in vitro. Biochim Biophys Acta 1778:1283-1291.

Nies AT, Koepsell H, Damme K, and Schwab M (2011) Organic cation transporters (OCTs, MATEs), in vitro and in vivo evidence for the importance in drug therapy. Handb Exp Pharmacol (201):105-167.

Noé J, Portmann R, Brun ME, and Funk C (2007) Substrate-dependent drug-drug interactions between gemfibrozil, fluvastatin and other organic anion-transporting peptide (OATP) substrates on OATP1B1, OATP2B1, and OATP1B3. Drug Metab Dispos 35:1308-1314.

Powell J, Farasyn T, Köck K, Meng X, Pahwa S, Brouwer KL, and Yue W (2014) Nove mechanism of impaired function of organic anion-transporting polypeptide 1B3 in human hepatocytes: post-translational regulation of OATP1B3 by protein kinase C activation. Drug Metab Dispos 42:1964-1970.

Roth M, Obaidat A, and Hagenbuch B (2012) OATPs, OATs and OCTs: the organic anion and cation transporters of the SLCO and SLC22A gene superfamilies. $\mathrm{Br} J$ Pharmacol $\mathbf{1 6 5}$ $1260-1287$.

Söderberg O, Gullberg M, Jarvius M, Ridderstråle K, Leuchowius KJ, Jarvius J, Wester K, Hydbring P, Bahram F, Larsson LG, et al. (2006) Direct observation of individual endogenous protein complexes in situ by proximity ligation. Nat Methods 3:995-1000.

Srimaroeng C, Cecile JP, Walden R, and Pritchard JB (2013) Regulation of renal organic anion transporter 3 (SLC22A8) expression and function by the integrity of lipid raft domains and their associated cytoskeleton. Cell Physiol Biochem 31:565-578.

Stagljar I, Korostensky C, Johnsson N, and te Heesen S (1998) A genetic system based on splitubiquitin for the analysis of interactions between membrane proteins in vivo. Proc Natl Acad Sc USA 95:5187-5192.

Stieger B and Hagenbuch B (2014) Organic anion-transporting polypeptides. Curr Top Membr 73: 205-232.

Stieger B, Hagenbuch B, Landmann L, Höchli M, Schroeder A, and Meier PJ (1994) In situ localization of the hepatocytic $\mathrm{Na}^{+} /$Taurocholate cotransporting polypeptide in rat liver. Gas troenterology 107:1781-1787.

Wang P, Wang WJ, Choi-Nurvitadhi J, Lescaille Y, Murray JW, and Wolkoff AW (2019) Rat organic anion transport protein $1 \mathrm{~A} 1$ interacts directly with organic anion transport protein $1 \mathrm{~A} 4$ facilitating its maturation and trafficking to the hepatocyte plasma membrane. Hepatology 70:2156-2170.

Yan H, Zhong G, Xu G, He W, Jing Z, Gao Z, Huang Y, Qi Y, Peng B, Wang H, et al. (2012) Sodium taurocholate cotransporting polypeptide is a functional receptor for human hepatitis B and D virus [published correction appears in eLife (2014) 3:e05570]. eLife 1:e00049.

Zhang L, Dresser MJ, Gray AT, Yost SC, Terashita S, and Giacomini KM (1997) Cloning and functional expression of a human liver organic cation transporter. Mol Pharmacol 51:913-921.

Zhang Y, Boxberger KH, and Hagenbuch B (2017) Organic anion transporting polypeptide 1B3 can form homo- and hetero-oligomers. PLoS One 12:e0180257.

Zhao W, Zitzow JD, Ehresman DJ, Chang SC, Butenhoff JL, Forster J, and Hagenbuch B (2015) $\mathrm{Na}^{+} /$Taurocholate cotransporting polypeptide and apical sodium-dependent bile acid transporter are involved in the disposition of perfluoroalkyl sulfonates in humans and rats. Toxicol Sci 146: $363-373$.

Address correspondence to: Bruno Hagenbuch, Department of Pharmacology, Toxicology and Therapeutics, The University of Kansas Medical Center, 3901 Rainbow Blvd., Kansas City, KS 66160. E-mail: bhagenbuch@kumc.edu 